\title{
Digestibilidad aparente de ingredientes de origen vegetal y animal en la cachama
}

\author{
Wálter Vásquez-Torres ${ }^{(1)}$, Martha Inés Yossa ${ }^{(1)}$ y Mariana Catalina Gutiérrez-Espinosa(1) \\ (1)Universidad de los Llanos, Instituto de Acuicultura (IALL), Km 12 Vía Puerto López, Villavicencio, Meta, Colombia. \\ E-mail: wvasquez@unillanos.edu.co, marthayossa@gmail.com, marianacgutierreze@gmail.com
}

\begin{abstract}
Resumen - El objetivo de esta investigación fue evaluar los coeficientes de digestibilidad aparente de materia seca (MS), proteína y energía de alimentos de origen animal y vegetal, utilizados en raciones para cachama (Piaractus brachypomus). Fueron formuladas 15 dietas experimentales, compuestas por $69,5 \%$ de una dieta referencia semipurificada, $0,5 \%$ de óxido de cromo y $30 \%$ del ingrediente a evaluar. En cada experimento, fueron utilizados 90 peces que se alimentaron durante cinco días con la correspondiente dieta; al quinto día, los animales fueron trasladados a tanques cónicos para recolección de heces. Los coeficientes de digestibilidad aparente (CDA) de proteína variaron de 92,1 a $84,7 \%$ entre los ingredientes proteicos de origen vegetal, de 85,0 a $68,5 \%$ en los proteicos de origen animal, y de 83,7 a 57,6\% entre los de origen vegetal con baja proteína. Los CDA de energía de torta de soya, gluten de maíz, harina de yuca integral y de todos los ingredientes de origen animal arrojaron valores superiores a 76\%. Los máximos CDA de MS variaron entre 71 y $78 \%$ y fueron observados en gluten, harina de yuca y en los ingredientes de origen animal. La cachama tiene alta capacidad para aprovechar eficientemente ingredientes de origen animal y vegetal.
\end{abstract}

Términos para indexación: Piaractus brachypomus, coeficientes de digestibilidad, energía digestible, ingredientes alimenticios, peces tropicales, proteína digestible.

\section{Apparent digestibility of ingredients from plants and animals in cachama}

Abstract - The objective of this work was to evaluate the apparent digestibility coefficients of dry matter (DM), protein and energy of ingredients from animals and plants utilized in the ration for cachama (Piaractus brachypomus). Fifteen experimental diets were formulated, which were composed by $69.5 \%$ of a semipurified reference diet, $0.5 \%$ chromium oxide, and $30 \%$ of the ingredient to be evaluated. In each experiment, 90 fish fed the corresponding diet were used; in the fifth day, fish were transferred to conical tanks for fecal sampling. The apparent digestibility coefficients (ADC) of protein varied between 92.1 and $84.7 \%$ among protein ingredients from plants, between 85.0 and $68.5 \%$ among protein ingredients from animals, and between 83.7 and $57.6 \%$ among low-protein ingredients from plants. Energy ADC of soybean meal, corn gluten, wholly yuca meal and of all ingredients from animals produced values higher than $76 \%$. The highest values for CDA of DM varied between 71 and $78 \%$ and were observed in gluten, yuca meal and in ingredients from animals. Cachama has a high ability to efficiently benefit from ingredients from animals and plants.

Index terms: Piaractus brachypomus, digestibility coeficients, digestible energy, feedstuffs, tropical fish, digestible protein.

\section{Introducción}

El valor nutricional de un alimento se basa en su composición química y en la capacidad del organismo que lo consume para digerir y absorber los nutrientes y la energía en él contenida (National Research Council, 1993). Sullivan \& Reigh (1995), Laining et al. (2003) y Köprücü \& Özdemir (2005) consideran que la combinación de conocimientos sobre composición química y digestibilidad de un ingrediente, a utilizar en la formulación de una ración, permite precisar los cálculos sobre su contribución en términos de nutrientes y de energía y, de igual manera, estimar la proporción no digerible que será eliminada con las heces; la formulación de dietas con base en estos dos criterios es la clave para lograr raciones nutricionalmente eficientes y de reducido impacto ambiental (Guillaume et al., 2004). El conocimiento de los coeficientes de digestibilidad viabiliza la inclusión de una gran variedad de productos y subproductos de la agroindustria en raciones para peces (Pezzato et al., 2002).

En la acuicultura moderna, rutinariamente, las dietas son formuladas con base en la digestibilidad aparente 
de los nutrientes y energía contenidos en las materias primas, prefiriéndose las que exhiben los mejores indicadores de digestibilidad (Cho \& Kaushik, 1990). Conforme lo destacaBureau etal.(1999), los coeficientes de digestibilidad aparente aportan información valiosa acerca de la disponibilidad de nutrientes y energía de las materias primas, necesarias para la formulación de raciones con niveles eficientes de nutrientes. Sklan et al. (2004) mostraron que la digestibilidad total de una ración es el producto de la sumatoria de los valores de digestibilidad de los nutrientes y de la energía presentes en los ingredientes que la componen. En este aspecto, la revisión de Glencross et al. (2007), sobre estrategias de evaluación de ingredientes en raciones para la acuicultura, dejó claro que, en términos de calidad, una ración será buena si los ingredientes que la componen también lo son.

La cachama (Piaractus brachypomus) es una especie nativa de los ríos de las cuencas del Orinoco y del Amazonas; tiene hábito omnívoro, con tendencia al consumo de frutas y semillas (Lucas, 2008) igual que el tambaqui (Colossoma macropomum) y el pacu (Piaractus mesopotamicus) (Araujo-Lima \& Goulding, 1997). A pesar de la importancia comercial de la cachama, en la literatura hay poca información sobre digestibilidad de materias primas para la especie. En países como Colombia, donde se cultiva comercialmente en estanques y jaulas, no existen raciones formuladas específicamente para cachama, razón por la cual se recurre al uso de concentrados diseñados para otras especies como tilapia o trucha. Sin embargo, estos concentrados no permiten a la cachama expresar todo su potencial de crecimiento, ya que no están formulados con base en las cualidades digestivas de esta especie. Sobre digestibilidad de ingredientes para este pez se reconocen las publicaciones de Torres-Agudelo \& Uribe-Hosie (1995), Fernandes et al. (2004) y Gutiérrez-Espinosa \& Vásquez-Torres (2008).

El objetivo de esta investigación fue evaluar y comparar entre si los coeficientes de digestibilidad aparente de materia seca (MS), proteína y energía de alimentos de origen animal y vegetal, utilizados en raciones para cachama (Piaractus brachypomus).

\section{Materiales y Métodos}

Esta investigación fue realizada en el Laboratorio Experimental de Alimentación y Nutrición de Peces
(LEANP) del Instituto de Acuicultura de la Universidad de los Llanos, Villavicencio (Meta), Colombia (44'24"N 7334'56" W).

Fueron evaluados los siguientes ingredientes: de origen vegetal, con alto contenido de proteína torta de soya, torta de girasol, gluten de maíz y soya integral; y energéticos - germen de maíz, harina de maíz amarillo americano, torta de palmiste, mezcla forrajera de maíz, harina de trigo duro o, harina de trigo de tercera, harina de arroz y yuca integral. También se evaluaron los seguintes ingredientes de origen animal: harinas pescado, de carne y huesos y de sangre. Como base de las dietas experimentales fue utilizada la dieta referencia semipurificada (DRS) desarrollada por Vásquez-Torres et al. (2002). Para cada ingrediente investigado fue elaborada una dieta compuesta por $69.5 \%$ de DRS, $30 \%$ del ingrediente en estudio y $0,5 \%$ de marcador inerte (óxido de cromo) (Cuadro 1). Las raciones fueron elaboradas en micro-extrusora (Exteec, Riberão Preto, SP, Brasil).

El análisis de nutrientes de las materias primas (Cuadro 2) indica que estas se corresponden con la composición de ingredientes de uso común en la industria de raciones balanceadas para peces en general (National Research Council, 1993; Guillaume et al., 2004).

Cuadro 1. Composición proximal de las dietas experimentales en cuanto a materia seca (MS), proteína bruta (PB), cenizas $(\mathrm{Cz})$ y energía bruta (EB).

\begin{tabular}{|c|c|c|c|c|}
\hline Dietas experimentales & $\begin{array}{l}\text { MS } \\
(\%)\end{array}$ & $\begin{array}{l}\text { PB } \\
---(\% \mathrm{I}\end{array}$ & $\begin{array}{c}\mathrm{Cz} \\
\mathrm{AS})---\end{array}$ & $\begin{array}{c}\text { EB } \\
\left(\mathrm{kcal} \mathrm{kg}^{-1}\right)\end{array}$ \\
\hline Dieta referencia semipurificada (DRS) & 70,7 & 30,9 & 4,2 & $4.695,1$ \\
\hline \multicolumn{5}{|c|}{ DRS con ingredientes proteicos de origen vegetal } \\
\hline Torta de soya & 81,2 & 39,3 & 5,3 & $4.583,2$ \\
\hline Soya integral & 78,7 & 34,6 & 4,4 & $4.917,9$ \\
\hline Torta de girasol & 82,9 & 34,5 & 5,5 & $4.704,8$ \\
\hline Gluten de maíz & 81,4 & 43,1 & 3,4 & $4.870,0$ \\
\hline \multicolumn{5}{|l|}{ DRS con ingredientes energéticos } \\
\hline Germen de maíz & 87,6 & 23,5 & 3,0 & $4.585,0$ \\
\hline Torta de palmiste & 92,2 & 29,4 & 3,7 & $4.780,8$ \\
\hline Harina de trigo de tercera & 80,4 & 29,1 & 4,3 & $4.522,2$ \\
\hline Harina de trigo duro (granillo) & 75,7 & 27,9 & 3,5 & $4.425,1$ \\
\hline Harina de arroz & 81,1 & 28,6 & 5,5 & $4.966,2$ \\
\hline Mezcla forrajera de maíz & 88,5 & 26,1 & 4,6 & $5.036,7$ \\
\hline Maíz amarillo americano & 73,4 & 23,5 & 3,3 & $4.531,2$ \\
\hline Harina de yuca integral & 74,3 & 23,4 & 4,3 & $4.355,6$ \\
\hline \multicolumn{5}{|l|}{ DRS con ingredientes de origen animal } \\
\hline Harina de pescado & 81,5 & 32,5 & 8,5 & $4.695,0$ \\
\hline Harina de carne y hueso & 78,7 & 36,7 & 5,7 & $5.052,3$ \\
\hline Harina de sangre & 83,5 & 41,2 & 3,8 & $5.118,8$ \\
\hline
\end{tabular}

Pesq. agropec. bras., Brasília, v.48, n.8, p.920-927, ago. 2013 DOI: 10.1590/S0100-204X2013000800016 
Se utilizaron seis tanques de concreto de $10 \mathrm{~m}^{2}$ (tanques de alimentación), nueve tanques cónicos de fibra de vidrio de $200 \mathrm{~L}$, con un dispositivo en el fondo para concentración y colecta de heces (sistema Guelph modificado) y dos tanques de $3 \mathrm{~m}$ de diámetro, para reposo de los peces entre pruebas; en todos los tanques se dispuso flujo de agua y aireación permanentes para mantener niveles de oxígeno superiores a $60 \%$ de saturación; la temperatura del agua fue $25,5 \pm 1,0^{\circ} \mathrm{C}$, el $\mathrm{pH}$ en $6,5 \pm 0,3$ y los niveles de amonio se mantuvieron en concentraciones inferiores a 0,2 ppm.

Fueron seleccionados 540 juveniles de cachama de $220 \mathrm{~g}$ de peso promedio; para cada ración a evaluar fue utilizado un grupo de 90 peces, los cuales fueron sometidos a un periodo de adaptación a la dieta experimental de cinco días, y el alimento fue suministrado hasta aparente saciedad, a las 9 y $16 \mathrm{~h}$. Al quinto día después de la ración matutina, los peces fueron transferidos de los tanques de alimentación a los tanques de colecta de heces, habiéndose colocado diez animales por tanque. A partir de la octava hora, tiempo de tránsito intestinal determinado en pruebas preliminares, se inició la colecta de heces. Durante 12 horas, con intervalos de una hora, fue retirado el material acumulado en el sedimentador; se eliminó el exceso de agua y se refrigeró el material para evitar procesos

Cuadro 2. Composición proximal de las materias primas investigadas en cuanto a materia seca (MS), proteína bruta $(\mathrm{PB})$, extracto etéreo $(\mathrm{EE})$, cenizas $(\mathrm{Cz})$ y energía bruta $(\mathrm{EB})$.

\begin{tabular}{|c|c|c|c|c|c|}
\hline Ingredientes & $\begin{array}{c}\text { MS } \\
(\%) \\
\end{array}$ & PB & $\begin{array}{c}\mathrm{EE} \\
(\% \mathrm{MS} \\
\end{array}$ & $\mathrm{Cz}$ & $\begin{array}{c}\text { EB } \\
\left(\mathrm{kcal} \mathrm{kg}^{-1}\right) \\
\end{array}$ \\
\hline \multicolumn{6}{|l|}{ Proteicos de origen vegetal } \\
\hline Torta de soya & 92,6 & 45,7 & 1,7 & 7,5 & $4.600,1$ \\
\hline Soya integral & 86,3 & 34,4 & 19,8 & 5,8 & $5.745,4$ \\
\hline Torta de girasol & 92,4 & 35,8 & 1,4 & 7,8 & $4.599,5$ \\
\hline Gluten de maíz & 91,7 & 57,7 & 2,1 & 1,8 & $5.546,1$ \\
\hline \multicolumn{6}{|l|}{ Energéticos } \\
\hline Germen de maíz & 89,7 & 19,5 & 8,5 & 1,1 & $5.102,2$ \\
\hline Torta de palmiste & 91,9 & 16,3 & 3,6 & 3,8 & $4.585,0$ \\
\hline Harina de trigo de tercera & 91,2 & 14,7 & 3,6 & 5,4 & $4.662,6$ \\
\hline Harina de trigo duro (granillo) & 88,8 & 12,1 & 1,9 & 1,8 & $4.213,9$ \\
\hline Harina de arroz & 90,1 & 12,4 & 14,4 & 8,0 & $4.762,8$ \\
\hline Mezcla forrajera de maíz & 92,1 & 11,5 & 14,1 & 5,3 & $4.768,0$ \\
\hline Maíz amarillo americano & 85,7 & 9,0 & 3,9 & 4,3 & $4.462,1$ \\
\hline Harina de yuca integral & 86,1 & 5,4 & 1,3 & 3,6 & $4.190,3$ \\
\hline \multicolumn{6}{|l|}{ Proteicos de origen animal } \\
\hline Harina de pescado & 93,8 & 58,4 & 9,8 & 21,5 & $4.875,2$ \\
\hline Harina de carne y hueso & 96,8 & 50,5 & 20,5 & 11,0 & $5.756,5$ \\
\hline Harina de sangre & 87,5 & 70,0 & 13,3 & 4,2 & $5.302,8$ \\
\hline
\end{tabular}

de descomposición. Para acopiar la cantidad suficiente de material fecal para los análisis de composición proximal y para determinación de óxido de cromo, las heces de tres tanques ( 30 peces) fueron reunidas en una sola muestra. De esta manera, se obtuvieron tres muestras que se asumieron como repeticiones de cada dieta experimental evaluada. Después del periodo de colecta los peces fueron trasladados a tanques de reposo, en donde permanecieron 12 días alimentados con la DRS hasta volver a ser utilizados para otra prueba diferente.

Los ingredientes, las dietas y las heces fueron analizados para determinar su composición proximal según metodologías estándar (Cunniff, 1995). El contenido de cromo en las dietas experimentales y en las heces se determinó por el método de digestión ácida. Los coeficientes de digestibilidad aparente (CDA) de ingredientes y de las dietas experimentales fueron determinados por el método indirecto (Cho et al., 1985), por la ecuación de digestibilidad aparente total (DAT) de las dietas, con DAT $(\%)=$ $100-100\left(\mathrm{Cr}_{\mathrm{DExp}} / \mathrm{Cr}_{\mathrm{H}}\right)$, y la ecuación del coeficiente de digestibilidad aparente de nutrientes $(\mathrm{N})$ y energía (E) de las dietas experimentales (Dexp) y de la DRS, con $\mathrm{CDA}=100-\left[100\left(\mathrm{Cr}_{\text {DExp }} / \mathrm{Cr}_{\mathrm{H}}\right)\left(\mathrm{N}_{\mathrm{H}} / \mathrm{N}_{\text {DExp }}\right)\right]$, en que: $\mathrm{Cr}_{\text {DExp }}=\%$ de $\mathrm{Cr}$ en la dieta experimental; $\mathrm{Cr}_{\mathrm{H}}=\%$ de $\mathrm{Cr}$ en las heces; $\mathrm{N}_{\mathrm{H}}=\%$ nutriente (o kcal kg-1 de energía) en las heces; $\mathrm{N}_{\text {DExp }}=\%$ de nutriente (o kcal kg-1 de energía) en la dieta experimental.

Los CDA de materia seca (MS), proteína (P) y energía (E) en cada ingrediente evaluado (\%CDA- $\left.\mathrm{N}_{\text {ing }}\right)$ fueron calculados con base en la digestibilidad de la DRS y de las dietas experimentales, por la ecuación propuesta por Bureau et al. (1999):

CDA-N $\mathrm{N}_{\text {ing }}=\left\{\left[(\mathrm{a}+\mathrm{b}) \mathrm{CDA}-\mathrm{N}_{\mathrm{DExp}}\right]-\left[\mathrm{a} \times \mathrm{CDA}-\mathrm{N}_{\mathrm{DRS}}\right] \mathrm{b}^{-1}\right\}$, en que: a es el aporte de nutriente de la DRS al contenido de nutriente de la dieta experimental y se calculó como a $=(\%$ de nutriente en DRS $)(100-i)$; $\mathrm{i}=\%$ de nutriente en la dieta combinada; $b$ es el aporte de nutriente del ingrediente en estudio al contenido de nutriente de la dieta experimental y se calculó como $\mathrm{b}=(\%$ de nutriente en DRS $)(\mathrm{i}) ;(\mathrm{a}+\mathrm{b})=\%$ de nutriente en la dieta experimental; CDA- $\mathrm{N}_{\mathrm{DExp}}=\mathrm{CDA}$ del nutriente en la dieta experimental; $\mathrm{CDA}-\mathrm{N}_{\mathrm{DRS}}=$ CDA del nutriente en la DRS.

Se utilizó un diseño experimental completamente al azar para 15 tratamientos (dietas experimentales) 
por triplicado. Los valores medios de los CDA fueron sometidos a ANOVA de una vía $(\mathrm{p}<0,05)$; para el caso de significancia estadística entre tratamientos se aplicó el test de Tukey, a 5\% de probabilidad, para comparaciones entre todos los ingredientes y al interior de los grupos. Se utilizó programa Systat for Windows (ver. 10.2).

\section{Resultados y Discusión}

En la evaluación de ingredientes para uso en raciones para la acuicultura, los aspectos que se investigan para definir su potencial nutricional son, entre otros, composición química, palatabilidad, presencia de antinutrientes y digestibilidad de sus nutrientes (Glencross et al., 2007).

Con respecto a la digestibilidad de la materia seca (MS), los valores más altos, $72,7 \%$ y superiores, se presentaron en los materiales de origen animal (Cuadro 3). Entre los ingredientes de origen vegetal, se obtuvieron valores del mismo orden solo para gluten de maíz y harina integral de yuca ( $p>0,05)$. Los CDAde las materias primas energéticas fueron menores $(p<0,05)$ $\mathrm{y}$, de estos, los más bajos se observaron en la mezcla forrajera de maíz y en la harina de trigo de tercera. La mayor parte de los coeficientes de digestibilidad de proteína (CDA-PB) fueron superiores a $75 \%$. La digestibilidad de ingredientes ricos en proteína, normalmente, oscila entre 75 y 95\% (National Research Council, 1993). En este experimento, los CDA-PB de los ingredientes proteicos vegetales, estadísticamente iguales entre sí, fueron superiores a 84,7\% ( $p>0,05)$, en tanto que, en las de origen animal, solamente la harina de pescado exhibió un valor semejante. En los ingredientes energéticos, que contenían proteína entre 5,4 y 19,5\% (Cuadro 2), los mayores coeficientes $(\mathrm{p}<0,05)$ se observaron en la torta de palmiste, las harinas de trigo duro y de yuca y el germen de maíz, con diferencias entre sí que no fueron estadísticamente significativas $(p>0,05)$. La biodisponibilidad de energía fue variable entre ingredientes y dentro de los grupos conformados en esta investigación ( $p<0,05)$; los coeficientes más altos, entre 76,6 y $84 \%$, se observaron en harina de pescado, de carne y hueso, de sangre, torta de soya, e igualmente en yuca integral; las diferencias entre sí no fueron estadísticamente significativas $(\mathrm{p}>0,05)$. El valor más bajo fue el de harina de trigo

Cuadro 3. Coeficientes de digestibilidad aparente (CDA) y contenidos digestibles de materia seca (MS D), proteína (PD) y energía (ED) de los ingredientes investigados para cachama.

\begin{tabular}{|c|c|c|c|c|c|c|}
\hline \multirow[t]{2}{*}{ Ingredientes } & \multicolumn{2}{|c|}{ Materia Seca } & \multicolumn{2}{|c|}{ Proteína } & \multicolumn{2}{|c|}{ Energía } \\
\hline & CDA $(\%)$ & MS D (kcal kg-1) & CDA $(\%)$ & PD (kcal kg-1) & CDA $(\%)$ & $\mathrm{ED}\left(\mathrm{kcal} \mathrm{kg}^{-1}\right)$ \\
\hline Dieta referencia semipurificada (DRS) & 74,07 & - & 92,95 & - & 80,57 & - \\
\hline \multicolumn{7}{|l|}{ Proteicos de origen vegetal } \\
\hline Torta de soya & $62,5 \pm 0,6 \mathrm{bB}^{(1)}$ & 57,9 & $92,1 \pm 0,7 \mathrm{aA}$ & 42,1 & $79,3 \pm 3,0 \mathrm{aA}$ & 3647,9 \\
\hline Soya integral & $44,2 \pm 1,8 \mathrm{eC}$ & 38,1 & $84,8 \pm 1,4 \mathrm{aA}$ & 29,2 & $54,6 \pm 2,5 \mathrm{cB}$ & 3137,0 \\
\hline Torta de girasol & $52,3 \pm 0,4 \mathrm{fD}$ & 48,3 & $84,9 \pm 3,5 \mathrm{aA}$ & 30,4 & $44,7 \pm 1,2 \mathrm{dC}$ & 2056,0 \\
\hline Gluten de maíz & $71,1 \pm 0,9 \mathrm{aA}$ & 65,2 & $84,7 \pm 1,9 \mathrm{aA}$ & 48,9 & $75,8 \pm 1,9 \mathrm{aA}$ & 4353,7 \\
\hline \multicolumn{7}{|l|}{ Energéticos } \\
\hline Germen de maíz & $55,3 \pm 3,8 \mathrm{cB}$ & 49,6 & $81,5 \pm 1,2 \mathrm{bB}$ & 15,8 & $53,3 \pm 3,7 \mathrm{cC}$ & 2719,5 \\
\hline Torta de palmiste & $57,5 \pm 2,3 \mathrm{bcB}$ & 52,8 & $90,6 \pm 2,2 \mathrm{abA}$ & 14,8 & $47,7 \pm 3,7 \mathrm{dC}$ & 2187,0 \\
\hline Harina de trigo de tercera & $48,5 \pm 0,3 \mathrm{eC}$ & 44,2 & $57,6 \pm 7,9 \mathrm{eD}$ & 8,5 & $32,9 \pm 4,3 \mathrm{eD}$ & 1534,0 \\
\hline Harina de trigo duro (granillo) & $53,5 \pm 3,3 \mathrm{cdB}$ & 47,3 & $86,4 \pm 1,2 \mathrm{abA}$ & 10,5 & $67,1 \pm 0,9 \mathrm{bcB}$ & 2827,5 \\
\hline Harina de arroz & $59,8 \pm 1,1 \mathrm{cB}$ & 53,9 & $75,0 \pm 3,6 \mathrm{bB}$ & 9,3 & $66,0 \pm 1,5 \mathrm{bcB}$ & 3143,4 \\
\hline Mezcla forrajera de maíz & $32,2 \pm 1,8 \mathrm{fD}$ & 29,7 & $74,6 \pm 1,2 \mathrm{bB}$ & 8,6 & $44,8 \pm 0,4 \mathrm{dC}$ & 2136,1 \\
\hline Maíz amarillo americano & $59,9 \pm 0,1 \mathrm{cdB}$ & 51,3 & $69,0 \pm 1,0 \mathrm{dC}$ & 6,2 & $57,6 \pm 7,0 \mathrm{cC}$ & 2570,2 \\
\hline Harina de yuca integral & $74,8 \pm 2,2 \mathrm{aA}$ & 64,4 & $83,7 \pm 3,4 \mathrm{abA}$ & 4,5 & $79,9 \pm 2,2 \mathrm{aA}$ & 3348,0 \\
\hline \multicolumn{7}{|l|}{ Proteicos de origen animal } \\
\hline Harina de pescado & $76,2 \pm 1,1 \mathrm{aA}$ & 71,5 & $85,0 \pm 0,3 \mathrm{abA}$ & 49,6 & $84,0 \pm 2,3 \mathrm{aA}$ & 4095,2 \\
\hline Harina de carne y hueso & $72,7 \pm 1,0 \mathrm{aA}$ & 66,7 & $68,5 \pm 1,1 \mathrm{dC}$ & 34,6 & $76,6 \pm 0,3 \mathrm{aB}$ & 4409,5 \\
\hline Harina de sangre & $78,6 \pm 3,7 \mathrm{aA}$ & 68,8 & $77,8 \pm 3,9 \mathrm{bB}$ & 54,5 & $78,2 \pm 2,9 \mathrm{aB}$ & 4146,8 \\
\hline
\end{tabular}

${ }^{(1)}$ Letras minúsculas diferentes, en la misma columna, indican significancia estadística a 5\% de probabilidad, en la comparación entre todos los ingredientes; letras mayúsculas diferentes, en la misma columna, indican diferencias entre ingredientes dentro de cada grupo, a 5\% de probabilidad. Los valores representan la media de tres repeticiones \pm desviación estándar (DE). 
de tercera, con $32,9 \%$, y el más alto el de harina de pescado, con $84,0 \%(\mathrm{p}<0,05)$.

El análisis individual de materias primas mostró que el gluten de maíz, ingrediente con la mayor proporción de proteína (57\%) entre los de origen vegetal, exhibió CDA de $84,7 \%$ para proteína y $78,5 \%$ para energía; estos valores fueron inferiores a los observados por Abimorad et al. (2008) en pacu $(95,6$ y 86,0\%). Este ingrediente es un subproducto desecado de la almidonería de maíz, constituido principalmente por el gluten obtenido durante la separación del almidón; además de la proteína, contiene entre 8 y $10 \%$ de fibra y aceites con alta proporción de ácidos grasos insaturados.

La torta de soya es uno de los ingredientes más usados en acuicultura, debido a que exhibe alta biodisponibilidad de proteína para peces en general; en la literatura, se reportan valores de digestibilidad entre 75,9 y $81,1 \%$ para cachama (Fernandes et al., 2004; Gutiérrez-Espinosa \& Vásquez-Torres, 2008), de 83,5 a 90,6\% para pacu, Piaractus mesopotamicus (Abimorad \& Carneiro, 2004; Abimorad et al., 2008), y de 87,4 a $92,4 \%$ para tilapia, Oreochromis sp. (Gonçalves \& Carneiro, 2003; Vásquez-Torres et al., 2010). Para carnívoros como dorado, Salminus brasilienses, se encontráron valores de digestibilidad de 93,6\% (Borghesi et al., 2009) y, para truchas y salmónidos en general, de 90,1 y 93,0\% (Sugiura et al., 1998). En esta investigación, el CDA-PB fue comparable con los más altos observados en los trabajos citados, mientras que el CDA-ED fue intermedio con respecto a valores descritos en esas investigaciones, en las cuales osciló entre $71,4 \%$ en tilapia (Boscolo et al., 2002) y 92,7\% en pacu (Abimorad \& Carneiro, 2004). La soya integral y la torta de girasol exhibieron alta biodisponibilidad de proteína, y sus coeficientes fueron semejantes entre sí con valores cercanos a $85 \%$; los CDA-ED obtenidos para estos dos ingredientes fueron bajos, comparados con los referidos para tilapia, por Sklan et al. (2004) y Vásquez-Torres et al. (2010), quienes reportaron valores entre 73,9 y $76,8 \%$ en soya y entre 65,5 y $80,4 \%$ en girasol. A pesar del alto contenido de proteína en estos ingredientes, los bajos coeficientes de digestibilidad de materia seca observados limitarían su uso en raciones para la especie, debido a que aproximadamente un $50 \%$ de lo que se utilice no podrá ser digerido.
Los CDA-MS de los ingredientes de origen animal fueron los más elevados $(\mathrm{p}<0,05)$, con un valor promedio de $75,8 \%$, aceptable con respecto a resultados en otras especies. El CDA-PB de la harina de pescado resultó ser 5\% más bajo que el observado por Fernandes et al. (2004) en cachama, quien obtuvo $90,5 \%$ y $7 \%$ más alto que el CDA-ED de 77,0\%. En pacu, Abimorad \& Carneiro (2004) obtuvieron CDA-PB y E de 88,4 y $78,1 \%$, y Abimorad et al. (2008) de 84,6 y 74,5. En tilapia, Pezzato et al. (2002) observaron coeficientes de $78,5 \%$ para proteína y de $80,1 \%$ para energía, y Guimarães et al. (2008), de 88,6\% para proteína; todos estos valores son equivalentes a los observados en este estudio.

La digestibilidad de la harina de carne y huesos fue $20 \%$ menor para proteína y cerca de $8 \%$ para energía, con respecto a los resultados descritos por Abimorad \& Carneiro (2004) para pacu. En comparación con datos para tilapia, reportados por Pezzato et al. (2002) y Guimarães et al. (2008), igualmente fueron menores en proporciones semejantes. Los CDA-PB y E de la harina de sangre, determinados en este estudio, fueron altos con respecto a los observados en diferentes especies. En pacu, Abimorad \& Carneiro (2004) obtuvieron 57,7\% para proteína y 67,4 para energía; en tilapia, Pezzato et al. (2002) observaron 50,7 y 89,4\% respectivamente para proteína y energía. Los estándares de calidad para harina de sangre, como ingrediente de raciones para peces, indican que esta debe contener entre 80 y $90 \%$ de proteína y bajas proporciones de grasa $(<1 \%)$ y de minerales (National Research Council, 1993). El ingrediente aquí evaluado tenía $70 \%$ de proteína, 13\% de grasa y 4,2\% de cenizas (Cuadro 2) e, igualmente, impurezas consistentes en pelos y otras partículas de naturaleza desconocida, aparentemente restos de cascos y cuernos; no era una materia prima de alta calidad, para que se pudieran justificar los altos coeficientes observados. Probablemente, los valores pudieron ser sobrestimados, y se presume, como causa probable de error, pérdida de nutrientes por lixiviación del bolo fecal que exhibió poca consistencia. De acuerdo con Bureau et al. (1999), la pérdida de material no digerido a partir de las heces es el principal factor causante de errores en los cálculos para la determinación de los CDA en organismos acuáticos; de otra parte, la digestibilidad de la harina de sangre y otros ingredientes de origen animal también puede variar, por diferencias en su calidad con respecto a composición, a procedencia de 
animales y procesos de obtención. Este último factor puede reducir en grado variable la digestibilidad, debido a que el tratamiento térmico requerido para la cocción y secado, durante su procesamiento, puede inducir cambios en las proteínas y producir daños a los aminoácidos y otros nutrientes.

La digestibilidad de los ingredientes energéticos, en general, fue baja, especialmente para materia seca y energía, mientras que para proteína fue mejor, con un promedio de $77,3 \%$. Los CDA-PB de palmiste, trigo duro y yuca integral presentaron valores que fluctuaron entre 83,7 y $90,6 \%$, semejantes a los observados en los ingredientes proteicos $(p>0,05)$. En relación a investigaciones con carácidos neotropicales, en Abimorad \& Carneiro (2004) y Fernandes et al. (2004), se reportaron CDA-PB de 84,3 y 93,9\% y de 83,1 y $82,4 \%$ para harina de maíz y trigo duro, respectivamente, mientras que para maíz Abimorad et al. (2008) observaron un coeficiente de $85,8 \%$, todos estos próximos en valor a los hallados en el presente estudio. En investigaciones realizadas con tilapias, los CDA-PB, reportados en seis trabajos consultados (Furuya et al., 2001; Pezzato et al., 2002; Gonçalves \& Carneiro, 2003; Sklan et al., 2004; Oliveira et al., 2007; Vásquez-Torres et al., 2010), variaron entre 64,2 y $93,4 \%$, con un promedio de $82,2 \%$ para maíz y de $81,2 \%$ para el trigo duro, promedio de valores entre 49,2 y $92,7 \%$. Los coeficientes de la harina de maíz en esos reportes fueron $6 \%$ mayores, y los de trigo duro $5 \%$ menores que los valores obtenidos en la actual investigación.

Para la harina de arroz, se observó un coeficiente similar al obtenido por Vásquez-Torres et al. (2010) en tilapia (79,3\%); sin embargo, para esta misma especie, Pezzato et al. (2002) y Gonçalves \& Carneiro (2003) obtuvieron resultados muy distantes, $44,2 \%$ y $94,8 \%$, respectivamente, posiblemente debido a diferencias en la calidad de las harinas utilizadas.

El germen de maíz o harina de germen de maíz desengrasada es un subproducto de la extracción del aceite del germen de maíz después de la molienda del grano por vía húmeda que, normalmente, exhibe entre 40 y $50 \%$ de fibra. Pezzato et al. (2002) reportaron CDA-MS y $\mathrm{PB}$ de $54,5 \%, 86,8 \%$ y 713 de 45,7 y $77,6 \%$, valores que fueron semejantes a los obtenidos en este estudio. Posiblemente su alto contenido en fibra es la causa de la baja disponibilidad total de este ingrediente para peces.
Para torta de palmiste, yucaintegraly mezcla forrajera de maíz, no se hallaron referencias en la literatura sobre su uso como ingrediente en raciones para peces. La torta de palmiste - un subproducto obtenido de la extracción física del aceite, a partir de las almendras del fruto de la palma de aceite -, es comercializada con destino a la alimentación de ganado bovino, aves y cerdos (Gómez et al., 2007); normalmente, contiene entre 14 y $17 \%$ de PB, 20 y 30\% de fibra, 7 y $10 \%$ de lípidos y hasta $47 \%$ de carbohidratos, proporciones que pueden variar en función del método de extracción. El contenido de proteína digestible de este ingrediente fue $13,8 \%$, valor que, comparativamente con lo que pueden aportar otros ingredientes energéticos evaluados, fue significativamente mayor $(\mathrm{p}<0,05)$.

Para la harina de yuca integral, que también es una materia prima usada en alimentación animal, principalmente de bovinos y cerdos, los CDA-PB y $\mathrm{E}$ aquí observados fueron notoriamente superiores con respecto a los descritos para otros ingredientes energéticos como el maíz, trigo y arroz; los resultados de este estudio indican que es viable considerarla, junto con el palmiste, como materias primas alternativas.

El salvado de maíz o mezcla forrajera de maíz es un subproducto obtenido del procesamiento del grano de maíz por la vía seca, luego de tamizar la harina después de la molienda; está compuesto por 55 a 70\% de fibra de las envolturas y capuchón y por 13 a $14 \%$ de material proveniente del grano. La digestibilidad de materia seca y de energía fueron menores de $50 \%$, y la disponibilidad de proteína fue semejante al promedio observado en los demás ingredientes energéticos.

Como se indicó anteriormente, en el ambiente natural, la dieta de la cachama básicamente está constituida por alimentos de origen vegetal, de los cuales hasta $98 \%$ corresponden a frutas y semillas. Sin embargo, durante la época de aguas bajas, cuando la disponibilidad de este tipo de alimentos es limitada, la especie se sustenta consumiendo pequeños peces e invertebrados, así como restos de animales muertos (Vásquez-Torres, 2005); su condición de pez omnívoro presupone una gran capacidad digestiva para aprovechar eficientemente la proteína proveniente de alimentos de diferente naturaleza, incluidos los de origen animal. Los resultados de esta investigación, en la que se observó que los CDA más altos se presentaron en los ingredientes proteicos, tanto de origen vegetal como animal, corroboran tal habilidad digestiva de la cachama.

Pesq. agropec. bras., Brasília, v.48, n.8, p.920-927, ago. 2013 DOI: 10.1590/S0100-204X2013000800016 


\section{Conclusiones}

1. La cachama tiene alta capacidad para aprovechar nutrientes contenidos en raciones elaboradas con ingredientes de origen vegetal y animal.

2. Los coeficientes de digestibilidad de la materia seca son más altos para ingredientes de origen animal.

3. La digestibilidad de la proteína fue superior a $81 \%$ en la mayor parte de los ingredientes investigados, excepto en las harinas de sangre, de carne y hueso y en las harinas de trigo, arroz, maíz y mezcla forragera de maíz.

4. La alta de digestibilidad del gluten de maíz indica que este ingrediente puede ser considerado una excelente fuente de proteína en dietas para cachama.

\section{Agradecimientos}

Al Instituto de Investigaciones de la Orinoquia Colombiana (IIOC) de la Universidad de los Llanos y al Ministerio de Agricultura y Desarrollo Rural, por la cofinanciación del proyecto a través del convenio 018-08 IICA-Unillanos; a la empresa colombiana de alimentos concentrados (Italcol), por el suministro de algunas materias primas.

\section{Referencias}

ABIMORAD, E.G.; CARNEIRO, D.J. Métodos de coleta de fezes e determinação dos coeficientes de digestibilidade da fração protéica e da energia de alimentos para o pacu, Piaractus mesopotamicus (Holmberg, 1887). Revista Brasileira de Zootecnia, v.33, p.1101-1109, 2004. DOI: 10.1590/S1516-35982004000500001.

ABIMORAD, E.G.; SQUASSONI, G.H.; CARNEIRO, D.J. Apparent digestibility of protein, energy, and amino acids in some selected feed ingredients for pacu Piaractus mesopotamicus. Aquaculture Nutrition, v.14, p.374-380, 2008. DOI: 10.1111/j.13 65-2095.2007.00544.x.

ARAUJO-LIMA, C.; GOULDING, M. So fruit a fish: ecology, conservation, and aquaculture of the amazon's tambaqui. New York: Columbia University, 1997. 191p.

BORGHESI, R.; DAIRIKI, J.K.; CYRINO, J.E.P. Apparent digestibility coefficients of selected feed ingredients for dourado Salminus brasiliensis. Aquaculture Nutrition, v.15, p.453-458, 2009. DOI: 10.1111/j.1365-2095.2008.00610.x.

BOSCOLO, W.R.; HAYASHI, C.; MEURER, F. Digestibilidade aparente da energia e nutrientes de alimentos convencionais e alternativos para a tilápia do nilo (Oreochromis niloticus, L.). Revista Brasileira de Zootecnia, v.31, p.539-545, 2002. DOI: 10.1590/S1516-35982002000300001.
BUREAU, D.P.; HARRIS, A.M.; CHO, C.Y. Apparent digestibility of rendered animal protein ingredients for rainbow trout (Oncorhynchus mykiss). Aquaculture, v.180, p.345-358, 1999. DOI: 10.1016/S0044-8486(99)00210-0.

CHO, C.Y.; COWEY, C.B.; WATANABE, T. Finfish nutrition in Asia: methodological approaches to research and development. Ottawa: IDRC, 1985. 154p.

CHO, C.Y.; KAUSHIK, S.J. Nutritional energetics in fish: energy and proteins utilization in rainbow trout (Salmo gairdneri). World Review of Nutrition and Dietetics, v.61, p.132-172, 1990.

CUNNIFF, P. Official methods of analysis of AOAC International. $16^{\text {th }}$ ed. Virgini: AOAC International, 1995. $2 \mathrm{v}$.

FERNANDES, J.B.K.; LOCHMANN, R.; BOCANEGRA, F.A. Apparent digestible energy and nutrient digestibility coefficients of diet ingredients for pacu Piaractus brachypomus. Journal of the World Aquaculture Society, v.35, p.237-244, 2004. DOI: 10.1111/j.1749-7345.2004.tb01080.x.

FURUYA, W.M.; PEZZATO, L.E.; PEZZATO, A.C.; BARROS, M.M.; CARVALHO, E.M. de. Coeficientes de digestibilidade e valores de aminoácidos digestíveis de alguns ingredientes para tilápia do nilo (Oreochromis niloticus). Revista Brasileira de Zootecnia, v.30, p.1143-1149, 2001. DOI: 10.1590/ S1516-35982001000500002.

GLENCROSS, B.D.; BOOTH, M.; ALLAN, G.L. A feed is only as good as its ingredients - a review of ingredient evaluation strategies for aquaculture feeds. Aquaculture Nutrition, v.13, p.17-34, 2007. DOI: 10.1111/j.1365-2095.2007.00450.x.

GÓMEZ, A.S.; BENAVIDES, C.I.; DIAZ, C.M. Evaluación de torta de palmiste (Elaeis guineensis) en alimentación de cerdos de ceba. Biotecnología en el sector agropecuario y agroindustrial, v.5, p.54-63, 2007.

GONÇALVES, E.G.; CARNEIRO, D.J. Coeficientes de digestibilidade aparente da proteína e energia de alguns ingredientes utilizados em dietas para o pintado (Pseudoplatystoma coruscans). Revista Brasileira de Zootecnia, v.32, p.779-786, 2003. DOI: 10.1590/S1516-35982003000400001.

GUILlAUME, J.; KAUSHIK, S.; BERGOT, P.; MÉTAILlER, R. Nutrición y alimentación de peces y crustáceos. Madrid: Mundi-Prensa, 2004. 475p.

GUIMARÃES, L.G.; PEZZATO, L.E.; BARROS, M.M. Amino acid availability and protein digestibility of several protein sources for Nile tilapia, Oreochromis niloticus. Aquaculture Nutrition, v.14, p.396-404, 2008. DOI: 10.1111/j.1365-2095.2007.00540.x.

GUTIÉRREZ-ESPINOSA， M.C.; VÁSQUEZ-TORRES, W. Digestibilidad de Glycine max L., soya, en juveniles de cachama blanca Piaractus brachypomus (Cuvier 1818). Orinoquia, v.12, p.141-148, 2008.

KÖPRÜCÜ, K.; ÖZDEMIR, Y. Apparent digestibility of selected feed ingredients for Nile tilapia (Oreochromis niloticus). Aquaculture, v.250, p.308-316, 2005. DOI: 10.1016/j. aquaculture.2004.12.003.

LAINING, A.; RACHMANSYAH; AHMAD, T.; WILLIAMS, K. Apparent digestibility of selected feed ingredients for humpback 
grouper, Cromileptes altivelis. Aquaculture, v.218, p.529-538, 2003. DOI: 10.1016/S0044-8486(02)00463-5.

LUCAS, C.M. Within flood season variation in fruit consumption and seed dispersal by two characin fishes of the Amazon. Bitropica, v.40, p.581-589, 2008. DOI: 10.1111/j.1744-7429.2008.00415.x.

NATIONAL RESEARCH COUNCIL. Nutrient requeriments of fish. Washington: NRC, 1993. 115p.

OLIVEIRA, G.R. de; LOGATO, P.V.R.; FREITAS, R.T.F. de; RODRIGUES, P.B.; FIALHO, E.T.; DIODATTI, F.C. Digestibilidade de nutrientes em ração com complexo enzimático para tilápia-do-nilo. Revista Brasileira de Zootecnia, v.36, p.1945-1952, 2007. DOI: 10.1590/S1516-35982007000900001.

PEZZATO, L.E.; MIRANDA, E.C. de; BARROS, M.M.; QUINTERO PINTO, L.G.; FURUYA, W.M.; PEZZATO, A.C. Digestibilidade aparente de ingredientes pela tilápia do nilo (Oreochromis niloticus). Revista Brasilera de Zootecnia, v.31, p.1595-1604, 2002. DOI: 10.1590/S1516-35982002000700001.

SKLAN, D.; PRAG, T.; LUPATSCH, I. Apparent digestibility coefficients of feed ingredients and their prediction in diets for tilapia Oreochromis niloticus x Oreochromis aureus (Teleostei, Cichlidae). Aquaculture Research, v.35, p.358-364, 2004. DOI: 10.1111/j.1365-2109.2004.01021.x.

SUGIURA, S.H.; DONG, F.M.; RATHBONE, C.K.; HARDY, R.W. Apparent protein digestibility and mineral availabilities in various feed ingredients for salmonid feeds. Aquaculture, v.159, p.177-2002, 1998. DOI: 10.1016/S0044-8486(97)00177-4.
SULLIVAN, J.A.; REIGH, R.C. Apparent digestibility of selected feedstuffs in diets for hybrid striped bass (Morone saxatilis $q \mathrm{x}$ Morone chrysops đ). Aquaculture, v.138, p.313-322, 1995. DOI: 10.1016/0044-8486(95)01071-8.

TORRES-AGUDELO, C.A.; URIBE-HOSIE, A. Evaluación de la digestibilidad aparente de cuatro subproductos agroindustriales, fuentes de proteína y energía, en la nutrición de la cachama blanca, Piaractus brachypomus Cuvier 1818. Boletim Científico INPA, v.3, p.40-65, 1995.

VÁSQUEZ-TORRES, W. A pirapitinga, reprodução e cultivo. In: BALDISSEROTTO, B.; GOMES, L. de C. (Ed.). Espécies nativas para piscicultura no Brasil. Santa Maria: UFSM, 2005. p.203-223.

VÁSQUEZ-TORRES, W.; PEREIRA-FILHO, M.; ARIAS-CASTELLANOS, J.A. Estudos para composição de uma dieta referência semipurificada para avaliação de exigências nutricionais em juvenis de pirapitinga, Piaractus brachypomus (Cuvier, 1818). Revista Brasileira de Zootecnia, v.31, p.283-292, 2002. DOI: 10.1590/S1516-35982002000200001.

VÁSQUEZ-TORRES, W.; YOSSA, M.I.; HERNÁNDEZ, G.; GUTIÉRREZ-ESPINOSA, M.C. Apparent digestibility of feed ingredients of common use in the balanced diets for tilapia red hybrid (Oreochromis sp.). Revista Colombiana de Ciencias Pecuarias, v.23, p.207-216, 2010.

Recibido en 2 de mayo de 2011 y aprobado en 21 de febrero de 2013 